\title{
Development of a Reading Material Recommender System Based On Design Science Research Approach
}

\author{
Evren Eryilmaz ${ }^{1}$, Brian Thoms ${ }^{2}$, Kuo-Hao Lee ${ }^{3}$, Melissa de Castro ${ }^{1}$ \\ ${ }^{1}$ Califronia State University Sacramento, ${ }^{2}$ Califronia State University Channel Islands, ${ }^{3}$ Bloomsburg University \\ evren.eryilmaz@csus.edu, brian.thoms@csuci.edu, klee@bloomu.edu, melissadecastro@csus.edu
}

\begin{abstract}
Using design science research (DSR), we outline the construction and evaluation of a recommender system incorporated into an existing computersupported collaborative learning environment. Drawing from Clark's communication theory and a user-centered design methodology, the proposed design aims to prevent users from having to develop their own conversational overload coping strategies detrimental to learning within large discussions. Two experiments were carried out to investigate the merits of three collaborative filtering recommender systems. Findings from the first experiment show that the constrained Pearson Correlation Coefficient (PCC) similarity metric produced the most accurate recommendations. Consistently, users reported that constrained PCC based recommendations served best to their needs, which prompted users to read more posts. Results from the second experiment strikingly suggest that constrained PCC based recommendations simplified users' navigation in large discussions by acting as implicit indicators of common ground, freeing users from having to develop their own coping strategies.
\end{abstract}

\section{Introduction}

Software development is a complex and challenging field. As noted by Robillard [1], software development is the "progressive crystallization of knowledge into a language that can be read and executed by a computer" (p.92). Accordingly, a principal challenge that lies at the heart of software development is knowledge asymmetry between those participants who possess business knowledge and developers who possess technical knowledge [2]. Collaboration can mitigate knowledge asymmetry by allowing team members to discuss and reconcile differing views, which can help identify errors early on in the software lifecycle and before they turn into software bugs in production environments.
Furthermore, as software projects become increasingly dispersed, whether as a result of outsourcing or open-source development models, collaboration is becoming more prevalent [3]. Thus, individuals completing degrees in technical fields must possess both strong technical knowledge and collaboration skills.

Collaborative learning is a pedagogical construct that plays a vital role in curriculum recommendations for information systems [4], computer science [5], and software engineering [6]. This pedagogical approach provides students with opportunities to discuss complex problems from multiple perspectives. Thus, the pedagogical strength of collaborative learning is its ability to capitalize on students' rigorous, coherent, engaging, and equitable discussions. There are numerous theories on how and why collaborative learning works, which are associated with group knowledge building, intersubjective meaning making, information exchange, conflict resolution, and participatory models (see Suthers [7]). In this sense, collaborative learning can bring students many benefits such as the development of collaboration skills, higher level thinking, agency, metacognition, and regulation [8]. Regarding software development, prior research has shown that collaborative learning can improve students' attitudes towards computer programming and help to decide if they can or should continue to study a technical field [9].

Computer-supported collaborative learning (CSCL) systems provide affordances for the production and continual improvement of ideas valuable to a community. Asynchronous online discussions (AODs) are popular CSCL tools often used in software development projects. AODs provide users with the time to prepare, reflect, and search for additional information before contributing to a discussion, allowing users to express more articulate ideas in written form. Moreover, the automatic preservation of discussion threads supports 
equitable discussions in the sense that users have the same opportunity to dig down, understanding issues and move a group into higher levels of thinking [10].

However, despite many affordances, collaboration is difficult to achieve. One reason considers conversational overload, which underscores users' feelings of being overwhelmed by a large number of discussion messages within the AOD [e.g., 11, 12]. To cope with conversational overload, users can resort to selective reading or skimming within the AOD. In one study, Peters \& Hewitt [12] showed that when feeling overwhelmed, users ignore discussion threads that do not interest them, skim long messages (i.e., 500 words or more) in order to look for items of interest, and skip messages written by some peers altogether. Drawing on these coping strategies, Qiu [13] reported that students from an online course skipped reading nearly half of all messages in an online discussion to save time. Under such conditions, students can miss relevant information that can aid in learning $[14,15]$.

To address these issues, this explorative study adopts a design science research (DSR) framework to design, construct, and evaluate a recommender system embedded into an AOD. DSR is similar to design-based research because they both focus on identification of a relevant problem, development and presentation of an artifact, evaluation to assess the artifact's utility, articulation of the value added to the knowledge base, and explanation of the implications. The goal of the proposed system is to prevent users from having to develop their own coping strategies detrimental to learning within large discussions.

The paper is organized as follows. The next section presents the theoretical background guiding this explorative study. We then apply the theoretical framework to develop a recommendation functionality incorporated into an asynchronous threaded online discussion system. Afterwards, we outline the research questions, methodology, and report the results. Finally, we conclude by discussing our findings and their implications.

\section{Theoretical background}

In large AODs, the effort users invest in dealing with messages can be a limiting factor for collaboration. The central premise behind conversational overload problem is that the effort required for explaining ideas in multiple and more connected ways is greater than the amount students are prepared to invest. For example, Eryilmaz et al.
[17] found that keeping an overview of an overwhelmingly large discussion (30 participants or more as defined by [18]) was too time-consuming for users and, thus, they failed to sustain gradual refinement of each other's ambiguous, figurative, and partial understandings.

Drawing on Clark's communication theory [19], we can consider the notion of common ground as a necessary ingredient for successful collaboration. Common ground refers to the goals, information, meaning, and ideas collaborators believe they share with each other [19]. Grounding is the process through which collaborators build common ground [19]. This theory has two important implications for our study.

First, common ground provides a context to carry out a task in ways that leverage the collective potential of a group. This is not to say group members must completely agree on each and every concept that they are dealing with. Rather, grounding lays a foundation for working effectively and efficiently in collaborative tasks, such as online collaborative literature processing. Different media can bring different affordances and constraints on grounding [15]. Within an AOD, users' grounding activities necessitate navigating large discussions to discern different perspectives as they refine their own views. This effort invested in navigation plays a catalytic role in grounding because the mere generation of a large number of brainstorming ideas, including the repetition of the same ideas may lead to an illusion of productivity.

A second important implication of Clark's communication theory for the purpose of our study is the principle of least collaborative effort. This principle underscores that conversational participants invest as little effort as necessary to achieve successful communication. Within educational AODs, students can be expected to apply the same economy of effort (e.g., "how do I complete the requirements of this collaborative learning task with the least amount of effort?") due to reasons of time pressure, task complexity, and indifference. However, what is sufficient to continue a conversation might not be sufficient for collaborative learning.

Students can implement a range of coping strategies in large discussions. On the one hand, students can increase their effort [11] or adopt new information management techniques (see [20]) to process a larger number of messages or digest long discussion threads. On the other hand, students can pay less attention to some messages, scan for points in a discussion where they can most easily contribute, or produce simpler or flawed responses [21, 22]. 
Adopting these counterproductive strategies can have deleterious effects on successful collaborative learning. Effective solutions for conversational overload include dividing a large group into smaller groups for discussion purposes (for a review of advantages and drawbacks of this solution see [13]), prescribing how students should interact via collaboration scripts (for a review of advantages and drawbacks of this solution see [15]), and utilizing recommender systems to analyze and filter information.

Recommender systems in AODs can reduce conversational overload in at least three ways. First, they can naturally facilitate common ground by emphasizing the presence of relevant posts. Second, more common ground on relevant posts means that keeping an overview of large discussions will become a less time consuming task. Third, reducing unnecessary navigation effort in large discussions can inhibit students from developing their own coping strategies.

\section{Artifact development}

Design Science Research (DSR) is a research paradigm that involves creating new knowledge through building and evaluating information technology artifacts [23, 24]. In this explorative study, the recommender system is the primary instantiation artifact. The overall objective of this artifact is to alleviate counterproductive coping strategies in AODs, which has shown to have deleterious effects on successful collaborative learning [e.g., 11, 12, 13].

The search for an effective instantiation artifact began with an examination of the four primary recommender systems categories [25]: collaborative filtering, content-based filtering, knowledge-based filtering, and hybrid approaches. Among these categories, collaborative filtering was chosen for three reasons. First, collaborative filtering fits well with social constructivism and provides explicit opportunities for students to interact with other likeminded students in order to diagnose and resolve common and pertinent problems of understanding. Second, explicit feedback (e.g., ratings) based on recommendations can be used as a fallback if no implicit feedback (e.g., posting behavior) is available [26]. Moreover, Abel et al. [26] found that a small amount of input data (two posts or two ratings) in a week is enough to generate precise recommendations while more input data do not generate better recommendations [26]. Third, as noted by Adomavicius \& Tuzhilin [27], collaborative filtering approaches do not depend on machine analysis of content, which is error-prone (e.g., deictic references invisible to a keyword metric), and they can make serendipitous recommendations.

Next, we adopted a user-centered design methodology (UCD) to define proper collaborative filtering based recommendations. The UCD focused on students' needs and the CSCL environment in which the recommendation functionality would be integrated [28]. Individual interviews with students enrolled in a systems development lifecycle course were conducted and identified their specific needs. These interviews gathered data along two main themes. The first theme identified that students' interests change over time depending on their level of understanding of a subject. A very important aspect of this theme is that an item recommended at a certain point in time could be too easy or too difficult for a student depending on the student's understanding of a subject. The second theme was that the system should be able to generate precise recommendations with little input data (e.g., using a ratings system). In other words, students perceived the rating activity to be time-consuming and demanding. Some even regarded the rating activity as the instructor's duty, rather than their own responsibility (for a similar finding see [29]). In this sense, the second theme essentially emphasizes the importance aforementioned in Abel et al.'s [26].

We incorporated our instantiation artifact into a modular, flexible, and extensible anchored discussion system first developed by Eryilmaz et al. [30]. In this CSCL environment, discussion threads are anchored to numbered and highlighted passages within the text to contextualize students' ideas. This distinct characteristic presents students with an intuitive means to collaboratively process academic literature. Prior research found that anchored AODs produce not only larger number of messages, but also longer discussion threads than regular forum discussions [18]. These findings further increase our chances of monitoring students' coping strategies in an anchored AOD system.

To the best of our knowledge, no anchored AOD was extended by a recommender system to improve students' online collaborative literature processing. However, prior research on regular forum discussions extended by recommendations based on collaborative filtering shows that a forum recommender improved students' summary writing ability, but not their learning motivation [31]. Similarly, Drachsler et al. [32] demonstrated that students who used a regular forum with a recommender system completed equal amounts of learning activities in less time than their counterparts who used a more traditional AOD. Moreover, Drachsler et al. [32] found that these more 
efficient students did not complete more learning activities than their counterparts.

\subsection{Recommender system}

An important design consideration was choosing a similarity metric. We developed three recommender systems to recommend students' annotated passages from reading materials. Collaborative filtering algorithms consider two users to be similar when they rate and agree on similar items. Pearson Correlation Coefficient (PCC) and Cosine Similarity are two popular similarity metrics used in collaborative filtering $[33,34]$. Our first recommender system implements the PCC, which calculates similarity by the following:

$$
\begin{aligned}
& \operatorname{sim}(a, b)=\frac{\sum_{p \in I}\left(r_{a, p}-\bar{r}_{a}\right)\left(r_{b, p}-\bar{r}_{b}\right)}{\sqrt{\sum_{p \in I}\left(r_{a, p}-\bar{r}_{a}\right)^{2}} \sqrt{\sum_{p \in I}\left(r_{b, p}-\bar{r}_{b}\right)^{2}}} \\
& a, b: \text { students } \\
& r_{a, p}: \text { rating of student a for message } \mathrm{p} \\
& I: \text { set of messages, rated both by a and } \mathrm{b}
\end{aligned}
$$

This metric considers only online discussion messages two students have rated, which can lead to spurious similarities between students who have rated only a few common messages. Cosine Similarity is a vector-space approach based on linear algebra rather than a statistical approach, uses all student ratings and is less likely to report spurious similarities [33, 34]. Therefore, our second recommendation functionality implements the cosine similarity metric, which calculates similarity by the following:

$$
\operatorname{Sim}(a, b)=\frac{\vec{r}_{a} \vec{r}_{b}}{\left\|\vec{r}_{a}\right\|\left\|\vec{r}_{b}\right\|}=\frac{\sum_{p} r_{a, p} r_{b, p}}{\sqrt{\sum_{p} r_{a, p}^{2}} \sqrt{\sum_{p} r_{b, p}^{2}}}
$$

Based on students' evaluation of these two recommender systems, we adopted a third approach, which implements the constrained PCC. This metric employs the median value in a rating scale to consider the impact of positive and negative ratings. For example, the median value $\left(r_{m e d}\right)$ in a scale from 1 to 5 is 3 . Since the scale of ratings is absolute, we know that values below 3 are negative, while values above 3 are positive. In this metric, similarity increases if two users rated an item both positively or both negatively. The constrained PCC calculates similarity by the following:

$$
\operatorname{sim}(a, b)=\frac{\sum_{p \in I}\left(r_{a, p}-r_{m e d}\right)\left(r_{b, p}-r_{m e d}\right)}{\left.\left.\sqrt{\sum_{p \in I}\left(r_{a, p}-r_{m e d}\right.}\right)^{2} \sqrt{\sum_{p \in I}\left(r_{b, p}-r_{m e d}\right.}\right)^{2}}
$$

In order to prevent the calculation of a high similarity score between two students who display coping strategies detrimental to learning, the instructor validated each similarity score individually. To make recommendations, similarity ratings of less than 0.1 are filtered out (for a similar approach see [35]). To take into account the change in students' interests over time, the system calculates unique similarity scores among students for each subject. In other words, similarity scores among students can change depending on their understanding of various subjects. Based on this similarity threshold, we used the weighted averaging mechanism for computing predictions because it is consistent with the social change theory [36] that deals with the preferences of individuals and of a group as a whole. The weighted averaging mechanism computes predictions as follows:

$$
\operatorname{Pred}(a, p)=\bar{r}_{\mathbf{a}}+\frac{\sum_{b \in N} \operatorname{sim}(a, b)\left(r_{b, p}-\bar{r}_{b}\right)}{\sum_{b \in N}|\operatorname{sim}(a, b)|}
$$

All three systems are constructed with the same user interface. Figure 1 displays the user interface of developed recommendation functionalities. The top of the left window pane displays personalized annotation recommendations to emphasize important points of interest. Clicking on an annotation recommendation highlights both the selected recommendation and relevant passage in red. Moreover, moving the cursor over an annotated passage keeps the annotated passage highlighted, while navigating the discussion to the pertinent thread and drawing a red border around that thread. This highlighting can be of great assistance to students who miss important messages because they are overwhelmed.

\subsection{Control system}

Aiming to isolate the effects of the recommendation functionality, we implemented a control version of the anchored discussion system developed in [30]. This control system highlights both the annotated text and the pertinent discussion thread when either element is under the cursor. Moreover, the control system includes the same rating scheme, but without the proposed recommender system. Figure 2 displays the user interface of the control system.

\section{Research questions}

As exploratory research, we aim to answer the following research questions: 
$\mathrm{R} 1$ : Is there any difference in the predictive accuracy and perceived usefulness of the developed recommender systems in online collaborative literature processing?

$\mathrm{R} 2$ : If $\mathrm{R} 1$, will the recommender system with the highest predictive accuracy and perceived usefulness decrease students' conversational overload coping strategies in online collaborative literature processing?

\section{Methodology}

Two experiments were conducted to answer the aforementioned research questions. The first experiment looked to determine if there was any difference in the predictive accuracy and perceived usefulness of the developed recommender systems.

We conducted the first experiment in a systems development lifecycle course required for information systems majors. Participants were 102 sophomore undergraduate students. We randomly divided students into three groups. Each group had 34 students. Each group was randomly assigned to a recommender system.

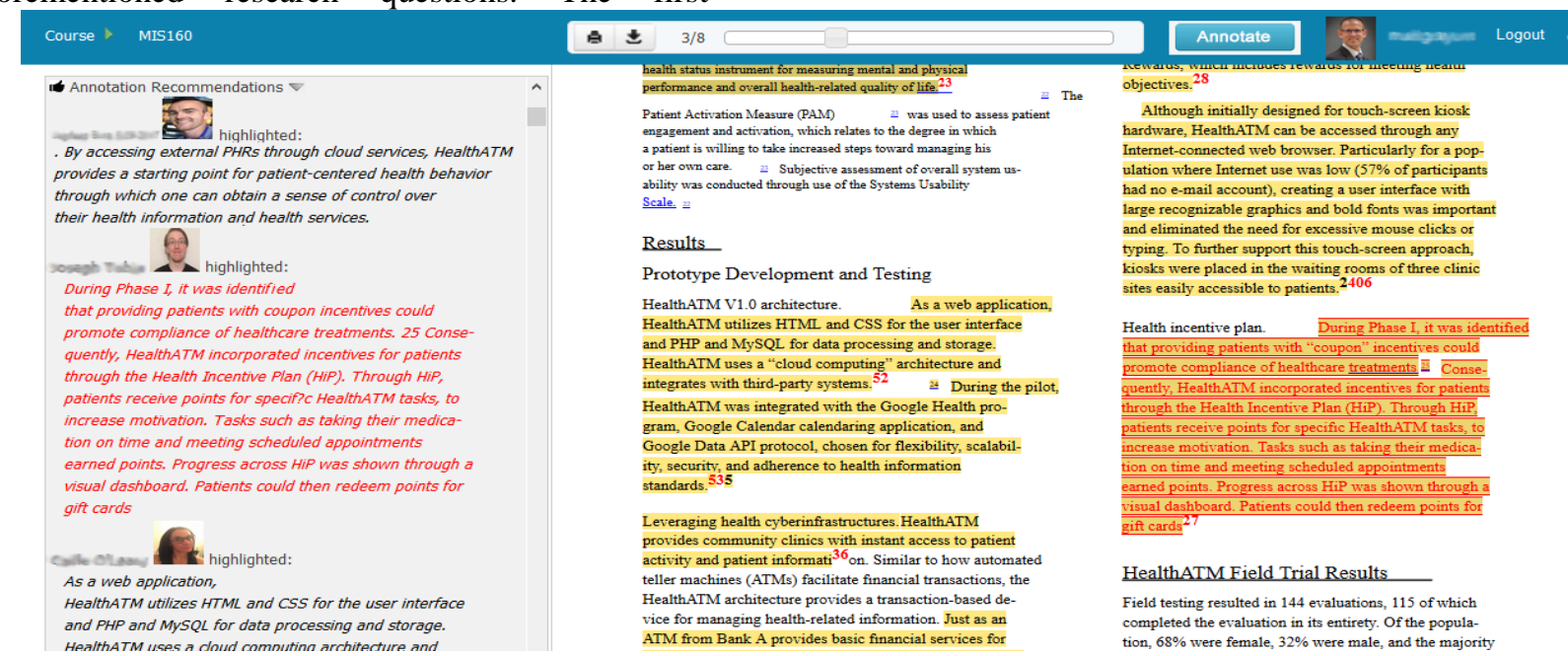

Figure 1. Recommendation functionality screenshot

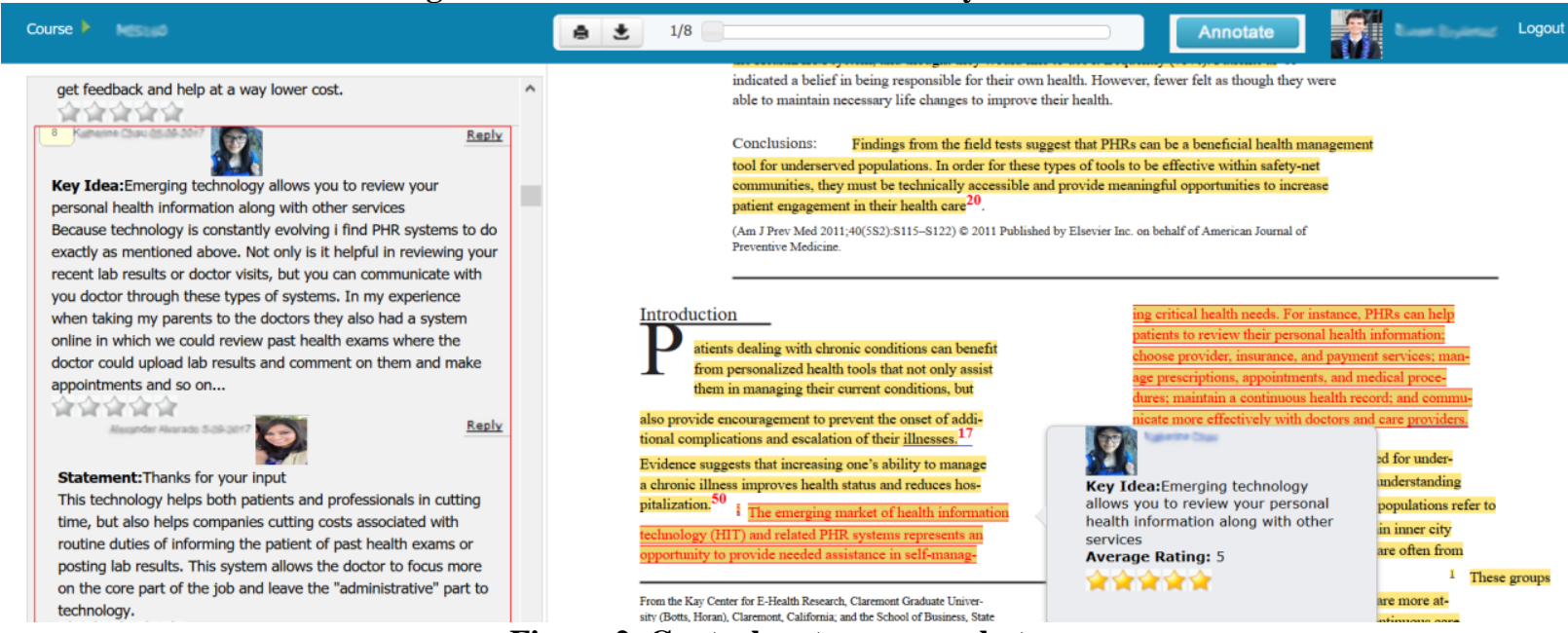

Figure 2. Control system screenshot

The instructional topic in the first experiment was personal health information systems. This topic included two research papers, which we arranged in the following sequence. Paper one was "HealthATM Personal Health Cyberinfrastructure for Underserved
Populations" [37]; and paper two was "Towards Intelligent Personal Health Record Systems: Review, Criteria and Extensions [38]. Each paper was covered during a two week online discussion period. At the end of the second discussion theme, we asked the participants to rate the quality of their 
recommendations and complete a perceived usefulness questionnaire.

The purpose of the second experiment was to determine if the recommender system with the highest predictive accuracy and perceived usefulness decreases users' coping strategies. We conducted the second experiment in two sections of an online human-computer interaction course. Participants were 64 undergraduate senior-level students majoring in information systems. The mean age of the participants was $22.43(S D=1.54)$. All participants were split into two sections of the same course. Each section had 32 students. Both sections were facilitated by the same instructor and followed the same schedule to eliminate confounding factors. We randomly assigned one section to the treatment group and the other to the control group. The treatment group had access to the recommender system with highest predictive accuracy and perceived usefulness, whereas the control group used the control software. The instructional topic for the purpose of the experiment was captology. This topic included two research papers, which we arranged in the following sequence. Paper one was "Creating Persuasive Technologies: an Eight-step Design Process [39]; and paper two was "Web Design Attributes in Building User Trust, Satisfaction, and Loyalty for a High Uncertainty Avoidance Culture [40]. Each paper was covered during a two-week online discussion period. At the end of the second discussion timeline, we asked participants to complete a coping strategies questionnaire.

All participants were required at minimum to make two annotations per paper and provide focused feedback as well as quality ratings to at least two fellow students' explanations for that paper. In order to facilitate a natural use of the recommender system, we merely offered it to the experimental group without requiring them to make use of it.

\subsection{Measuring predictive accuracy}

From a technical standpoint, measuring how close the recommender systems' predictions are to the true user ratings is a natural starting point for evaluating recommendations. Mean absolute error, normalized mean absolute error, and root mean squared error (RMSE) are three common measures for prediction accuracy [36]. Among these measures, we chose RMSE because it has the benefit of penalizing large errors by squaring the errors before they are averaged. Moreover, RMSE is in the same 5-star scale as the original ratings, which helps interpreting the results. Students were asked to rate the quality of the recommended annotations on a 5-star scale.

\subsection{Measuring perceived usefulness}

Next, we measured the perceived usefulness of our prototypes because a prototype might achieve high accuracy by only computing predictions for easy-to-predict items for which users are less likely to need computer-assisted predictions [25]. We adopted a questionnaire developed by Wang \& Yang [31]. The questionnaire included the following items: (1) "The recommendations were exactly what I was looking for"; (2) "I was surprised by the recommendations"; (3) "The recommendations helped me to read instructional materials more effectively"; (4) "The recommendations prompted me to read postings on the forum"; (5) "The recommendations prompted me to write on the forum". Students were asked to complete the questionnaire by using a five-point Likert-type scale ranging from 1 (strongly disagree) to 5 (strongly agree).

\subsection{Measuring conversational overload coping strategies}

Finally, we measured the coping strategies as reported by users. We adopted a questionnaire developed by Peters \& Hewitt [12]. The questionnaire included the following items: (1) "In an average week, what percentage of the week's notes do you read?", (2) "Of the notes you open, approximately what percentage of notes do you skim quickly or not read to the end?" Students were asked to complete the questionnaire by using a five-point scale ranging from $0-20 \%$ to $81-100 \%$.

\section{Results}

\subsection{Predictive accuracy results}

Table 1 displays the predictive accuracy results based on rating-prediction pairs from the first experiment. These scores suggest that the constrained PCC had the smallest penalty for large errors.

Table 1. Predictive accuracy results

\begin{tabular}{|c|c|}
\hline Similarity Metric & RMSE \\
\hline Pearson correlation coefficient (PCC) & 1.21 \\
\hline Cosine Similarity & 1.73 \\
\hline Constrained PCC & 0.87 \\
\hline
\end{tabular}

\subsection{Perceived usefulness results}

The Cronbach's alpha internal consistency value for the 5 items was 0.78 , indicating acceptable scale 
reliability. Table 2 shows statistically significant differences among the similarity metrics as determined by the $\mathrm{p}$-value corresponding to the Fstatistic of one-way ANOVA. Next, we conducted the Tukey HSD test to identify which specific similarity metrics differed as determined by the Tukey HSD p-value.

Table 2. Perceived usability results

\begin{tabular}{|c|c|c|c|c|c|}
\hline \multicolumn{6}{|c|}{ Questionnaire Item: "The recommendations were exactly what I was looking for" } \\
\hline Similarity Metric & $n$ & $M$ & $S D$ & $F$ & $p$ \\
\hline PCC & 34 & 4.06 & 0.60 & 12.90 & $<0.001 * * *$ \\
\hline Cosine Similarity & 34 & 3.62 & 0.36 & & \\
\hline Constrained PCC & 34 & 4.44 & 0.38 & & \\
\hline Contrast & $\begin{array}{c}\text { Tukey HSD } \\
\text { Q statistic }\end{array}$ & $\begin{array}{c}\text { Tukey HSD } \\
\text { p-value }\end{array}$ & & & \\
\hline Cosine Similarity vs PCC & 3.85 & $0.02 *$ & & & \\
\hline Cosine Similarity vs Constrained PCC & 3.33 & $0.05 *$ & & & \\
\hline PCC vs Constrained PCC & 7.18 & $0.001 * * *$ & & & \\
\hline \multicolumn{6}{|c|}{ Questionnaire Item: "I was surprised by the recommendations" } \\
\hline Similarity Metric & $n$ & $M$ & $S D$ & $F$ & $p$ \\
\hline PCC & 34 & 4.24 & 0.43 & 1.39 & 0.25 \\
\hline Cosine Similarity & 34 & 4.09 & 0.45 & & \\
\hline Constrained PCC & 34 & 4.35 & 0.42 & & \\
\hline \multicolumn{6}{|c|}{ Questionnaire Item: "The recommendations helped me to read instructional materials more effectively" } \\
\hline Similarity Metric & $n$ & $M$ & $S D$ & $F$ & $p$ \\
\hline PCC & 34 & 4.29 & 0.46 & 1.15 & 0.32 \\
\hline Cosine Similarity & 34 & 4.15 & 0.49 & & \\
\hline Constrained PCC & 34 & 4.38 & 0.31 & & \\
\hline \multicolumn{6}{|c|}{ Questionnaire Item: "The recommendations prompted me to read postings on the forum" } \\
\hline Similarity Metric & $n$ & $M$ & $S D$ & $F$ & $p$ \\
\hline PCC & 34 & 4.18 & 0.51 & 11.82 & $<0.001 * * *$ \\
\hline Cosine Similarity & 34 & 3.73 & 0.69 & & \\
\hline Constrained PCC & 34 & 4.59 & 0.37 & & \\
\hline Contrast & $\begin{array}{l}\text { Tukey HSD } \\
\text { Q statistic }\end{array}$ & $\begin{array}{c}\text { Tukey HSD } \\
\text { p-value }\end{array}$ & & & \\
\hline Cosine Similarity vs PCC & 3.56 & $0.04 *$ & & & \\
\hline Cosine Similarity vs Constrained PCC & 6.88 & $0.001 * * *$ & & & \\
\hline PCC vs Constrained PCC & 3.32 & $0.05 *$ & & & \\
\hline \multicolumn{6}{|c|}{ Questionnaire Item: "The recommendations prompted me to write on the forum" } \\
\hline Similarity Metric & $n$ & $M$ & $S D$ & $F$ & $p$ \\
\hline PCC & 34 & 4.09 & 0.26 & 3.53 & $0.03^{*}$ \\
\hline Cosine Similarity & 34 & 3.89 & 0.59 & & \\
\hline Constrained PCC & 34 & 4.26 & 0.20 & & \\
\hline Contrast & $\begin{array}{c}\text { Tukey HSD } \\
\text { Q statistic }\end{array}$ & $\begin{array}{c}\text { Tukey HSD } \\
\text { p-value }\end{array}$ & & & \\
\hline Cosine Similarity vs PCC & 2.02 & 0.33 & & & \\
\hline Cosine Similarity vs Constrained PCC & 3.76 & $0.02 *$ & & & \\
\hline PCC vs Constrained PCC & 1.73 & 0.44 & & & \\
\hline
\end{tabular}

Note. $d f$ between groups $=2 ;$ df within groups $=99 ; * p<0.05, * * p<0.01, * * * p<0.001$

\subsection{Conversational overload coping strategies results}

Based on results reported in Tables 1 and 2, we compared the recommender system with constrained
PCC with the control software in the second experiment. Table 3 presents conversational overload coping strategies as reported by the participants. 
Table 3. Conversational overload coping strategies results

\begin{tabular}{|l|c|c|c|c|c|c|}
\hline & \multicolumn{2}{|c|}{$\begin{array}{l}\text { Control } \\
\text { System }\end{array}$} & \multicolumn{2}{c|}{ Constrained PCC } & \multicolumn{2}{l|}{} \\
\hline $\begin{array}{l}\text { "In an average week, what } \\
\text { percentage of the week's notes do } \\
\text { you read?" }\end{array}$ & $\mathrm{f}$ & $\%$ & $\mathrm{f}$ & $\%$ & $z$ & $p$ \\
\hline $0-20 \%$ & 8 & 24 & 1 & 3 & 2.51 & $0.01^{* *}$ \\
\hline $21-40 \%$ & 5 & 15 & 4 & 12 & 0.36 & 0.72 \\
\hline $41-60 \%$ & 8 & 24 & 6 & 18 & 0.6 & 0.55 \\
\hline $61-80 \%$ & 8 & 24 & 17 & 50 & -2.26 & $0.02^{*}$ \\
\hline $81-100 \%$ & 5 & 15 & 6 & 18 & -0.33 & 0.74 \\
\hline $\begin{array}{l}\text { "Of the notes you open, } \\
\text { approximately, what percentage of } \\
\text { notes do you skim quickly or not } \\
\text { read the end?" }\end{array}$ & & & & & & \\
\hline $0-20 \%$ & 1 & 3 & 11 & & & \\
\hline $21-40 \%$ & 7 & 19 & 6 & 18 & 0.31 & 0.76 \\
\hline $41-60 \%$ & 14 & 41 & 6 & 18 & 2.13 & $0.03^{*}$ \\
\hline $61-80 \%$ & 10 & 31 & 3 & 9 & 4.66 & $0.04^{*}$ \\
\hline $81-100 \%$ & & & & & & \\
\hline
\end{tabular}

Note. $* p<0.05, * * p<0.01, * * * p<0.001$

\section{Discussion}

The aim of this design science research paper was to prevent users from having to develop their own coping strategies detrimental to learning within large discussions.

Regarding research question 1, predictive accuracy results show that the constrained PCC's RMSE was under 1.0, which means that most of the time this recommendation functionality's predictions agreed with users' ratings, or had a difference of 1 rank. Consistently, users reported that recommendations based on this similarity metric were closest to what they were looking for, and these recommendations prompted users to read postings on the forum. A possible explanation of these findings lies in the similarity computations [33, 34]. The Cosine Similarity metric used all student ratings for each topic when calculating a similarity score. This approach produced the least accurate recommendations. The PCC similarity metric improved the results by considering only messages both students had rated in a topic. Finally, the constrained PCC similarity metric clearly produced the best results by considering the impact of positive and negative ratings for each topic. We thus argue that navigating large discussions was a less timeconsuming task with the constrained PCC similarity metric. In contrast, there were no differences among the similarity metrics with respect to the following questionnaire items: "The recommendations helped me to read instructional materials more effectively"; "I was surprised by the recommendations". While these findings may seem to counter our explanation above, they empirically demonstrate that utilizing a recommender system when collaboratively discussing complex instructional topics within large discussions is better than having no recommendations at all. Interestingly, most participants in the first experiment were intrigued by the recommendations whether or not they were accurate. A possible explanation of this finding is that participants had never used a recommender system for online learning conversations.

Regarding research question 2, our findings are consistent with Qiu [13]. Detailed in Table 3, results suggests that the control group did not fully realize the constructivist affordances of the control system. Returning to Clark's Communication Theory, this symptom represents how control group quickly became overwhelmed and read only few messages as the number of messages grew. Strikingly, $50 \%$ of the treatment group reported they read $61-80 \%$ of weekly notes. Moreover, these users reported that they skimmed fewer notes than the control group. These findings suggest that recommendations simplified navigation in large discussions by acting implicit indicators of common ground on important topics of interest. Thus, students were freed from having to develop their own coping strategies. Overall, these findings extend prior research by Erdt et al. [25], who assert that little is still known about the way students perceive and react to recommendations. 
Finally, some limitations merit consideration. First, although pre-validated self-reporting measures are easy to administer, they are also subject to various biases. Future research aims to gather objective data through eye-tracking. Second, this study does not explicitly examine the quality of students' discussions and social capital they derived from those large discussions. Finally, we did not examine explicit grounding processes even if we conceptualized grounding as navigating large discussions to discern different perspectives. Future research will focus on examining users' awareness of who has the same understanding problem with them; who has a different view about an understanding problem; and who has potential to assist them solving an understanding problem. Despite these limitations, this paper opens up new research avenues to create more unobtrusive, effective, and adaptive CSCL systems.

\section{References}

[1] Robillard, P. N., "The Role of Knowledge in Software Development", Communications of the ACM 1999, 42(1), pp. 87-92.

[2] Benaroch, M., Lichtenstein, Y., \& Fink, L., "Contract Design Choices and the Balance of Ex-Ante and Ex-Post Transaction Costs in Software Development Outsourcing. MIS Quarterly 2015, 40(1), pp. 57-82.

[3] Kudaravalli, S., Faraj, S., \& Johnson, S. L., “A Configural Approach to Coordinating Expertise in Software Development Teams", MIS Quarterly 2017, 41(1), pp.4364.

[4] Topi, H., Valacich, J. S., Wright, R. T., Kaiser, K., Nunamaker Jr, J. F., Sipior, J. C., \& de Vreede, G. J., IS "2010: Curriculum Guidelines for Undergraduate Degree Programs in Information Systems", Communications of the Association for Information Systems 2010, 26(1), pp. 359428 .

[5] Joint Task Force on Computing Curricula IEEE Computer Society Association for Computing Machinery (ACM), "Curriculum Guidelines for Undergraduate Degree Programs in Software Engineering", Retrieved from http://www.acm.org/binaries/content/assets/education/se20 14.pdf

[6] Joint Task Force on Computing Curricula Association for Computing Machinery (ACM) and IEEE Computer Society, "Curriculum Guidelines for Undergraduate Degree Programs in Computer Science", Retrieved from http://www.acm.org/education/CS2013-final-report.pdf

[7] Suthers, D. D., "Technology Affordances for Intersubjective Meaning Making: A Research Agenda for
CSCL", International Journal of Computer-Supported Collaborative Learning 2006, 1(3), pp. 315-337.

[8] Erkens, M., Bodemer, D., \& Hoppe, H. U., "Improving Collaborative Learning in the Classroom: Text Mining Based Grouping and Representing", International Journal of Computer-Supported Collaborative Learning 2016, 11(4), pp. 387-415.

[9] Berland, M., Davis, D., \& Smith, C. P., "AMOEBA: Designing for Collaboration in Computer Science Classrooms Through Live Learning Analytics", International Journal of Computer-Supported Collaborative Learning 2015, 10(4), pp. 425-447.

[10] Scardamalia, M. \& Bereiter, C., "Knowledge Building: Theory, Pedagogy, and Technology", In R. K. Sawyer (Ed.), The Cambridge Handbook of the Learning Sciences 2006, pp.97-115. Cambridge University Press.

[11] Jones, Q., Ravid, G., \& Rafaeli, S., "Information Overload and the Message Dynamics of Online Interaction Spaces: A Theoretical Model and Empirical Exploration", Information systems research 2004, 15(2), pp 194-210.

[12] Peters, V. L., \& Hewitt, J., "An Investigation of Student Practices in Asynchronous Computer Conferencing Courses", Computers \& Education 2010, 54(4), pp. 951961.

[13] Qiu, M., \& McDougall, D., "Influence of Group Configuration on Online Discourse Reading", Computers \& Education 2015, 87, pp. 151-165.

[14] Abel, F., Bittencourt, I. I., Costa, E., Henze, N., Krause, D., \& Vassileva, J., "Recommendations in Online Discussion Forums for E-learning Systems", IEEE Transactions on Learning Technologies 2010, 3(2), pp.165176.

[15] Dillenbourg, P., \& Betrancourt, M., "Collaboration Load", Handling Complexity in Learning Environments: Theory and Research 2006, pp. 141-165.

[16] van der Pol, J., Admiraal, W., \& Simons, P. R. J., "Context Enhancement for Co-intentionality and Coreference in Asynchronous CMC", AI \& Society 2006, 20(3), pp. 301-313.

[17] Eryilmaz, E., van der Pol, J., Ryan, T., Clark, P. M., \& Mary, J., "Enhancing Student Knowledge Acquisition from Online Learning Conversations", International Journal of Computer-Supported Collaborative Learning 2013, 8(1), pp. 113-144.

[18] Hiltz, S. R. Impacts of College-level Courses via Asynchronous Learning Networks: Some Preliminary Results", Journal of Asynchronous Learning Networks 1997, 1(2), pp. 1-19. 
[19] Clark, H.H., \& Brennan S.E., "Grounding in Communication", In L. Resnick, J. Levine \& S. Teasley (Eds.), Perspectives on Socially Shared Cognition 1991, Hyattsville, MD: American Psychological Association pp. 127-149.

[20] Wise, A. F., Hausknecht, S. N., \& Zhao, Y., "Attending to Others' Posts in Asynchronous Discussions: Learners' Online "Listening" and its Relationship to Speaking”, International Journal of Computer-Supported Collaborative Learning 2014, 9(2), pp. 185-209.

[21] Ridings, C., \& Wasko, M., "Online Discussion Group Sustainability: Investigating the Interplay Between Structural Dynamics and Social Dynamics Over Time", Journal of the Association for Information Systems 2010, 11(2), pp. 95-121.

[22] Eppler, M. J., \& Mengis, J., "The Concept of Information Overload: A Review of Literature from Organization Science, Accounting, Marketing, MIS, and Related disciplines", "The Information Society 2004, 20(5), pp. 325-344.

[23] Hevner, A. R., March, S. T., Park, J., \& Ram, S., "Design Science in Information Systems Research", MIS Quarterly 2004, 28(1), pp. 75-105.

[24] Gregor, S., \& Hevner, A. R., "Positioning and Presenting Design Science Research for Maximum Impact", MIS Quarterly 2013, 37(2), pp. 337-355.

[25] Erdt, M., Fernandez, A., \& Rensing, C., "Evaluating Recommender Systems for Technology Enhanced Learning: A Quantitative Survey", IEEE Transactions on Learning Technologies 2015, 8(4), pp. 326-344.

[26] Abel, F., Bittencourt, I. I., Costa, E., Henze, N., Krause, D., \& Vassileva, J., "Recommendations in Online Discussion Forums for E-learning Systems", IEEE Transactions on Learning Technologies 2010, 3(2), pp. 165-176.

[27] Adomavicius, G., \& Tuzhilin, A., "Toward the Next Generation of Recommender Systems: A Survey of the State-Of-TheArt and Possible Extensions", IEEE Transactions on Knowledge and Data Engineering 2005, 17(6), pp. 734-749.

[28] Gulliksen, J., Göransson, B., Boivie, I., Blomkvist, S., Persson, J., \& Cajander, A., "Key Principles for UserCentered Systems Design", Behaviour and Information Technology 2003, 22(6), pp. 397-409.

[29] Ballantyne, R., Hughes, K., \& Mylonas, A., "Developing Procedures for Implementing Peer Assessment in Large Classes Using an Action Research Process", Assessment \& Evaluation in Higher Education 2002, 27(5), pp. 427-441.

[30] Eryilmaz, E., Thoms, B., Canelon, J., "How Design Science Research Helps Improving Learning Efficiency in
Online Conversations", Communications of the Association of Information Systems 2018, 42(21).

[31] Wang, P. Y., \& Yang, H. C., "Using Collaborative Filtering to Support College Students' Use of Online Forum for English Learning", Computers \& Education 2012, 59(2), pp. 628-637.

[32] Drachsler, H., Hummel, H. G., Van den Berg, B., Eshuis, J., Waterink, W., Nadolski, R., Berlanga, A., Boers, N., \& Koper, R., "Effects of the ISIS Recommender System for Navigation Support in Self-Organised Learning Networks", Educational Technology \& Society 2009, 12(3), pp. 115-126.

[33] Liu, H., Hu, Z., Mian, A., Tian, H., \& Zhu, X., “A New User Similarity Model to Improve the Accuracy of Collaborative Filtering", Knowledge-Based Systems 2014, 56, pp. 156-166.

[34] Ludford, P. J., Cosley, D., Frankowski, D., \& Terveen, L., "Think Different: Increasing Online Community Participation Using Uniqueness and Group Dissimilarity", In Proceedings of the SIGCHI Conference on Human Factors in Computing Systems 2004, pp. 631-638.

[35] Thoms, B., "A Dynamic Social Feedback System to Support Learning and Social Interaction in Higher Education", IEEE Transactions on Learning Technologies 2011, 4(4), pp. 340-352.

[36] Ekstrand, M. D., Riedl, J. T., \& Konstan, J. A., "Collaborative Filtering Recommender Systems", Foundations and Trends ${ }^{\circledR}$ in Human-Computer Interaction 2011, 4(2), pp. 81-173.

[37] Botts, N. E., Horan, T. A., \& Thoms, B. P., "HealthATM: Personal Health Cyberinfrastructure for Underserved Populations", American Journal of Preventive Medicine 2011, 40(5), pp. S115-S122.

[38] Genitsaridi, I., Kondylakis, H., Koumakis, L., Marias, K., \& Tsiknakis, M., "Towards Intelligent Personal Health Record Systems: Review, Criteria and Extensions", Procedia Computer Science 2013, 21, pp. 327-334.

[39] Fogg, B. J., "Creating Persuasive Technologies: An Eight-step Design Process", In Proceedings of the 4th International Conference on Persuasive Technology 2009, ACM.

[40] Faisal, C. N., Gonzalez-Rodriguez, M., FernandezLanvin, D., \& de Andres-Suarez, J., "Web Design Attributes in Building User Trust, Satisfaction, and Loyalty for a High Uncertainty Avoidance Culture", IEEE Transactions on Human-Machine Systems 2017, 47(6), pp. 847-859. 